\title{
Enema Powder Dosage Form
}

National Cancer Institute

\section{Source}

National Cancer Institute. Enema Powder Dosage Form. NCI Thesaurus. Code C64885.

A powder that is suspended in liquid prior to administration to the rectum. 